\title{
A quasi shanker distribution and its applications
}

\begin{abstract}
In the present paper, a two-parameter quasi Shanker distribution (QSD) which includes one parameter Shanker distribution introduced by Shanker ${ }^{1}$ as a special case has been proposed. Its statistical and mathematical properties including moments and moments based measures, hazard rate function, mean residual life function, stochastic ordering, mean deviations, Bonferroni and Lorenz curves and stress-strength reliability have also been discussed. The method of maximum likelihood estimation has been discussed for estimating the parameters of QSD. Finally, the goodness of fit of the QSD has been discussed with two real lifetime data and the fit is quite satisfactory over one parameter exponential, Lindley and Shanker distributions.
\end{abstract}

\author{
Volume 6 Issue I - 2017
}

\section{Rama Shanker}

Department of Statistics, Eritrea Institute of Technology, Eritrea

Correspondence: Rama Shanker, Department of Statistics, Eritrea Institute of Technology, Asmara, Eritrea, Email shankerrama2009@gmail.com, kkshukla22@gmail.com

Received: June 03,2017 | Published: June 13, 2017

Keywords: shanker distribution, moments, hazard rate function, mean residual life function, stochastic ordering, mean deviations, stress-strength reliability, estimation of parameters, goodness of fit.

\section{Introduction}

Shanker ${ }^{1}$ has introduced a one parameter lifetime distribution for modeling lifetime data from biomedical science and engineering having probability density function(pdf) and cumulative distribution function(cdf) given by

$$
\begin{aligned}
& f_{1}(x ; \theta)=\frac{\theta^{2}}{\theta^{2}+1}(\theta+x) e^{-\theta x} ; x>0, \theta>0 \\
& F_{1}(x, \theta)=1-\left[1+\frac{\theta x}{\theta^{2}+1}\right] e^{-\theta x} ; x>0, \theta>0
\end{aligned}
$$

Shanker ${ }^{1}$ has shown that it gives better fit than both one parameter exponential and Lindley ${ }^{2}$ distributions. This distribution is a mixture of exponential $(\theta)$ and gamma $(2, \theta)$ distributions with their mixing proportion $\frac{\theta^{2}}{\theta^{2}+1}$ and $\frac{1}{\theta^{2}+1}$ respectively.

The first four moments about origin of Shanker distribution obtained by Shanker ${ }^{1}$ are given as

$$
\begin{aligned}
& \mu_{1}^{\prime}=\frac{\theta^{2}+2}{\theta\left(\theta^{2}+1\right)}, \mu_{2}^{\prime}=\frac{2\left(\theta^{2}+3\right)}{\theta^{2}\left(\theta^{2}+1\right)}, \mu_{3}^{\prime}=\frac{6\left(\theta^{2}+4\right)}{\theta^{3}\left(\theta^{2}+1\right)}, \\
& \mu_{4}^{\prime}=\frac{24\left(\theta^{2}+5\right)}{\theta^{4}\left(\theta^{2}+1\right)}
\end{aligned}
$$

The central moments of Shanker distribution obtained by Shanker are

$$
\begin{gathered}
\mu_{2}=\frac{\theta^{4}+4 \theta^{2}+2}{\theta^{2}\left(\theta^{2}+1\right)^{2}} \\
\mu_{3}=\frac{2\left(\theta^{6}+6 \theta^{4}+6 \theta^{2}+2\right)}{\theta^{3}\left(\theta^{2}+1\right)^{3}} \\
\mu_{4}=\frac{3\left(3 \theta^{8}+24 \theta^{6}+44 \theta^{4}+32 \theta^{2}+8\right)}{\theta^{4}\left(\theta^{2}+1\right)^{4}}
\end{gathered}
$$

Shanker ${ }^{1}$ studied its important properties including coefficient of variation, skewness, kurtosis, Index of dispersion, hazard rate function, mean residual life function, stochastic ordering, mean deviations, order statistics, Bonferroni and Lorenz curves, Renyi entropy measure, and stress-strength reliability. The discrete Poisson - Shanker distribution, a Poisson mixture of Shanker distribution has also been studied by Shanker. ${ }^{3}$

Recall that the Lindley distribution, introduced by Lindley ${ }^{2}$ in the context of Bayesian analysis as a counter example of fiducial statistics, is defined by its pdf and cdf

$$
\begin{aligned}
& f_{2}(x ; \theta)=\frac{\theta^{2}}{\theta+1}(1+x) e^{-\theta x} ; x>0, \theta>0 \\
& F_{2}(x ; \theta)=1-\left[1+\frac{\theta x}{\theta+1}\right] e^{-\theta x} ; x>0, \theta>0
\end{aligned}
$$


In this paper, a two - parameter quasi Shanker distribution (QSD), of which one parameter Shanker distribution introduced by Shanker ${ }^{1}$ is a particular case, has been proposed. Its raw moments and central moments have been obtained and coefficients of variation, skewness, kurtosis and index of dispersion have been discussed. Some of its important mathematical and statistical properties including hazard rate function, mean residual life function, stochastic ordering, mean deviations, Bonferroni and Lorenz curves and stress-strength reliability have also been discussed. The estimation of the parameters has been discussed using maximum likelihood estimation. The goodness of fit of QSD has been illustrated with two real lifetime data sets and the fit has been compared with one parameter exponential, Lindley and Shanker distributions.

\section{A quasi shanker distribution}

A two - parameter quasi Shanker distribution (QSD) having parameters $\theta$ and $\alpha$ is defined by its pdf

$f(x ; \theta, \alpha)=\frac{\theta^{3}}{\theta^{3}+\theta+2 \alpha}\left(\theta+x+\alpha x^{2}\right) e^{-\theta x} ; x>0, \theta>0, \theta^{3}+\theta+2 \alpha>0$.

It can be easily verified that (2.1) reduces to the Shanker distribution (1.1) at $\alpha=0$. It can be easily verified that QSD is a three-component mixture of exponential $(\theta)$, gamma $(2, \theta)$ and gamma $(3, \theta)$ distributions. We have
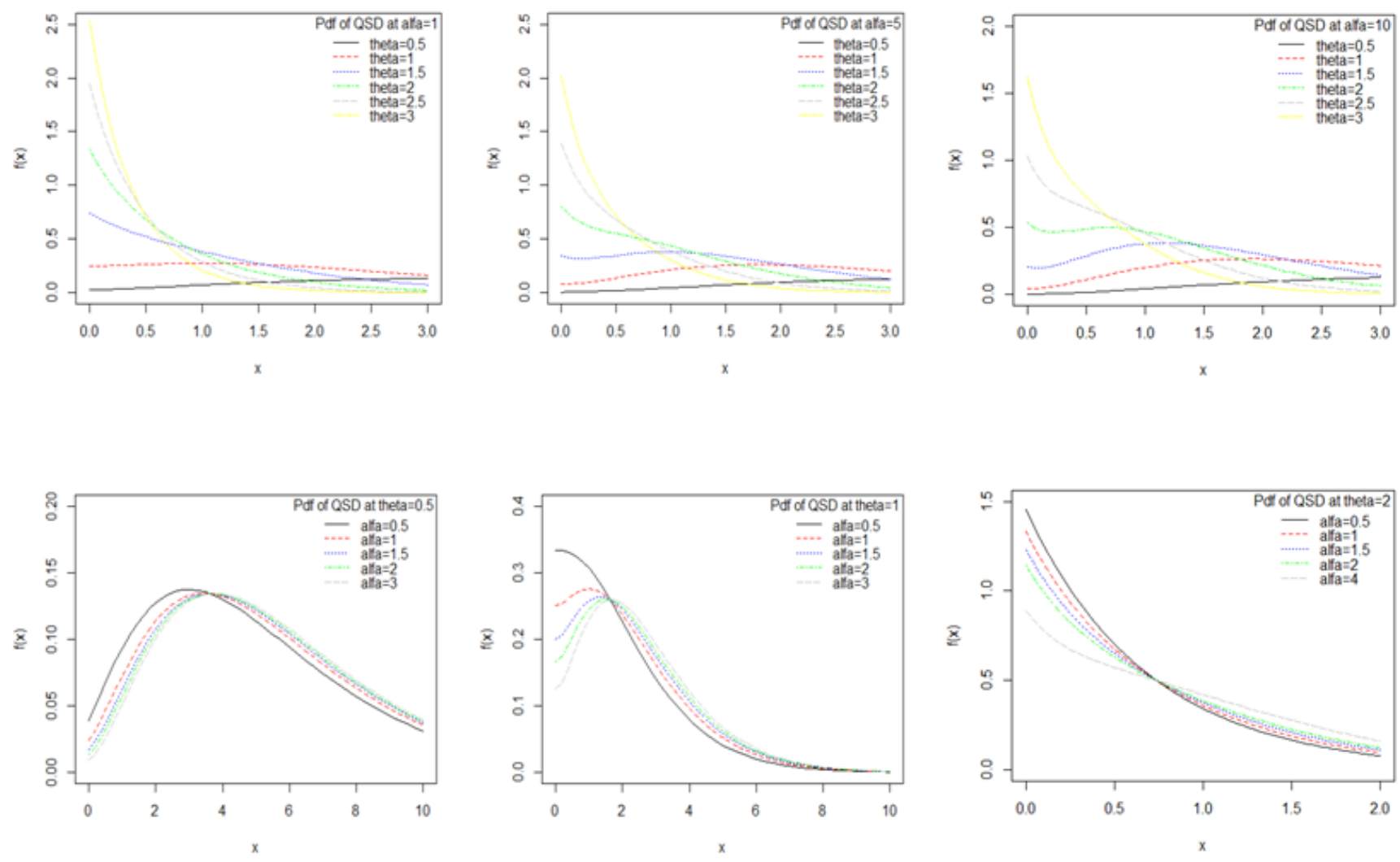

where

$$
\begin{gathered}
p_{1}=\frac{\theta^{3}}{\theta^{3}+\theta+2 \alpha}, p_{2}=\frac{\theta}{\theta^{3}+\theta+2 \alpha}, \\
f_{1}(x ; \theta)=\theta e^{-\theta x} ; x>0, \theta>0 \\
f_{2}(x ; 2, \theta)=\frac{\theta^{2}}{\Gamma(2)} e^{-\theta x} x^{2-1} ; x>0, \theta>0 \\
f_{3}(x ; 3, \theta)=\frac{\theta^{3}}{\Gamma(3)} e^{-\theta x} x^{3-1} ; x>0, \theta>0
\end{gathered}
$$

The corresponding cdf of QSD (2.1) can be obtained as

$$
F(x ; \theta, \alpha)=1-\left[1+\frac{\alpha \theta^{2} x^{2}+\theta x(\theta+2 \alpha)}{\theta^{3}+\theta+2 \alpha}\right] e^{-\theta x} ; x>0, \theta>0
$$

The nature and behavior of the pdf and the cdf of QSD for varying values of the parameters $\theta$ and $\alpha$ have been explained graphically and presented in Figures $1 \& 2$, respectively. $f(x ; \theta, \alpha)=p_{1} f_{1}(x ; \theta)+p_{2} f_{2}(x ; 2, \theta)+\left(1-p_{1}-p_{2}\right) f_{3}(x ; 3, \theta)(2$

Figure I Graphs of the pdf of QSD for varying values of parameters $\theta$ and $\alpha$. 

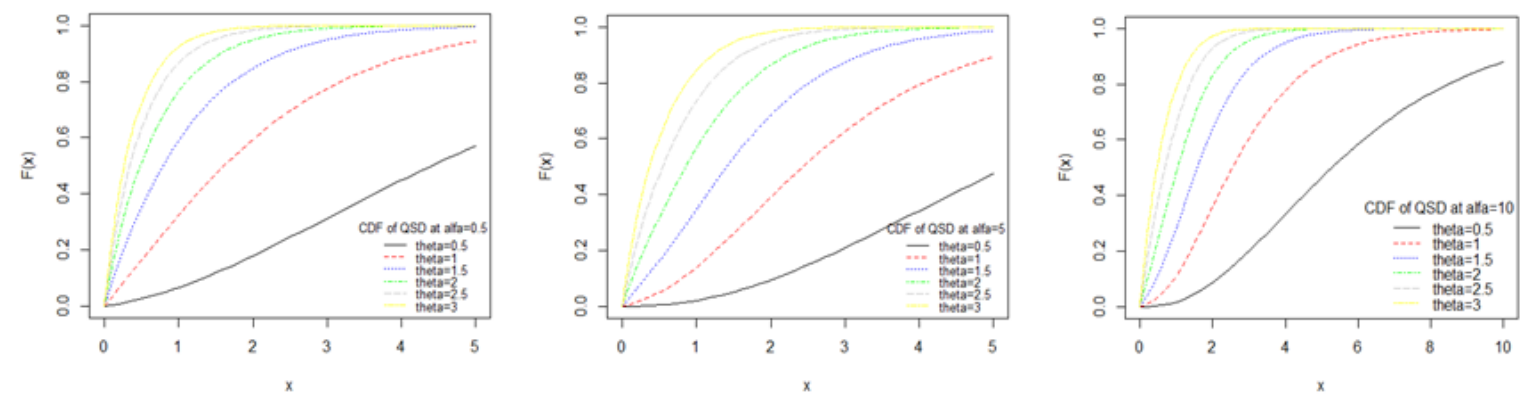

Figure 2 Graphs of the cdf of QSD for varying values of parameters $\theta$ and $\alpha$.

\section{Statistical constants}

The $r$ th moment about origin of QSD can be obtained as

$$
\mu_{r}^{\prime}=\frac{r !\left[\theta^{3}+(r+1) \theta+(r+1)(r+2) \alpha\right]}{\theta^{r}\left(\theta^{3}+\theta+2 \alpha\right)} ; r=1,2,3, . .
$$

Thus, the first four moments about origin of QSD are given by

$$
\begin{gathered}
\mu_{1}^{\prime}=\frac{\theta^{3}+2 \theta+6 \alpha}{\theta\left(\theta^{3}+\theta+2 \alpha\right)}, \mu_{2}^{\prime}=\frac{2\left(\theta^{3}+3 \theta+12 \alpha\right)}{\theta^{2}\left(\theta^{3}+\theta+2 \alpha\right)} \\
\mu_{3}=\frac{2\left\{\theta^{9}+6 \theta^{7}+30 \theta^{6} \alpha+6 \theta^{5}+42 \theta^{4} \alpha+\left(36 \alpha^{2}+2\right) \theta^{3}+18 \theta^{2} \alpha+36 \theta \alpha^{2}+24 \alpha^{3}\right\}}{\theta^{3}\left(\theta^{3}+\theta+2 \alpha\right)^{3}} \\
\mu_{4}=\frac{3\left\{\begin{array}{l}
3 \theta^{3}+24 \theta^{10}+128 \theta^{9} \alpha+44 \theta^{8}+344 \theta^{7} \alpha+\left(408 \alpha^{2}+32\right) \theta^{6}+320 \theta^{5} \alpha \\
\left.+\left(768 \alpha^{2}+8\right) \theta^{4}+\left(576 \alpha^{3}+96 \alpha\right) \theta^{3}+336 \theta^{2} \alpha^{2}+480 \theta \alpha^{3}+240 \alpha^{4}\right\}
\end{array}\right.}{\theta^{4}\left(\theta^{3}+\theta+2 \alpha\right)^{4}}
\end{gathered}
$$

Using relationship between central moments and moments about origin, the central moments of QSD (2.1) are thus obtained as

$$
\mu_{2}=\frac{\theta^{6}+4 \theta^{4}+16 \theta^{3} \alpha+2 \theta^{2}+12 \theta \alpha+12 \alpha^{2}}{\theta^{2}\left(\theta^{3}+\theta+2 \alpha\right)^{2}}
$$

The coefficient of variation $(C . V)$, coefficient of skewness $\left(\beta_{2}\right)$, coefficient of kurtosis $\left(\beta_{2}\right)$ and index of dispersion $(\gamma)$ of QSD are obtained as

$$
\begin{gathered}
C . V=\frac{\sigma}{\mu_{1}^{\prime}}=\frac{\sqrt{\theta^{6}+4 \theta^{4}+16 \theta^{3} \alpha+2 \theta^{2}+12 \theta \alpha+12 \alpha^{2}}}{\theta^{3}+2 \theta+6 \alpha} \\
\sqrt{\beta_{1}}=\frac{\mu_{3}}{\mu_{2}^{3 / 2}}=\frac{2\left\{\theta^{9}+6 \theta^{7}+30 \theta^{6} \alpha+6 \theta^{5}+42 \theta^{4} \alpha+\left(36 \alpha^{2}+2\right) \theta^{3}+18 \theta^{2} \alpha+36 \theta \alpha^{2}+24 \alpha^{3}\right\}}{\left(\theta^{6}+4 \theta^{4}+16 \theta^{3} \alpha+2 \theta^{2}+12 \theta \alpha+12 \alpha^{2}\right)^{3 / 2}} \\
\beta_{2}=\frac{\mu_{4}}{\mu_{2}{ }^{2}}=\frac{3\left\{\begin{array}{l}
3 \theta^{12}+24 \theta^{10}+128 \theta^{9} \alpha+44 \theta^{8}+344 \theta^{7} \alpha+\left(408 \alpha^{2}+32\right) \theta^{6}+320 \theta^{5} \alpha \\
+\left(768 \alpha^{2}+8\right) \theta^{4}+\left(576 \alpha^{3}+96 \alpha\right) \theta^{3}+336 \theta^{2} \alpha^{2}+480 \theta \alpha^{3}+240 \alpha^{4}
\end{array}\right\}}{\left(\theta^{6}+4 \theta^{4}+16 \theta^{3} \alpha+2 \theta^{2}+12 \theta \alpha+12 \alpha^{2}\right)^{2}}
\end{gathered}
$$




$$
\gamma=\frac{\sigma^{2}}{\mu_{1}^{\prime}}=\frac{\theta^{6}+4 \theta^{4}+16 \theta^{3} \alpha+2 \theta^{2}+12 \theta \alpha+12 \alpha^{2}}{\theta\left(\theta^{3}+\theta+2 \alpha\right)\left(\theta^{3}+2 \theta+6 \alpha\right)}
$$

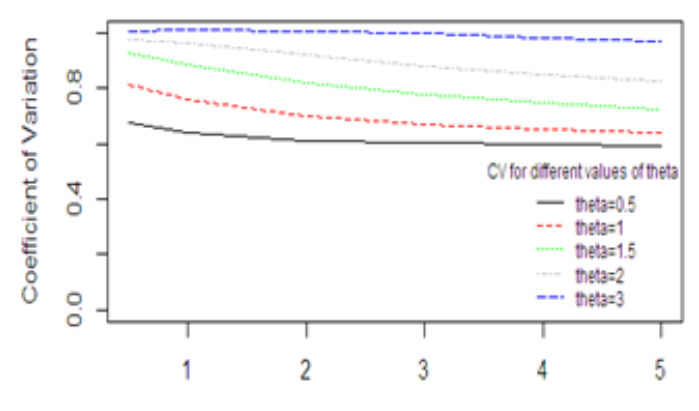

alpha

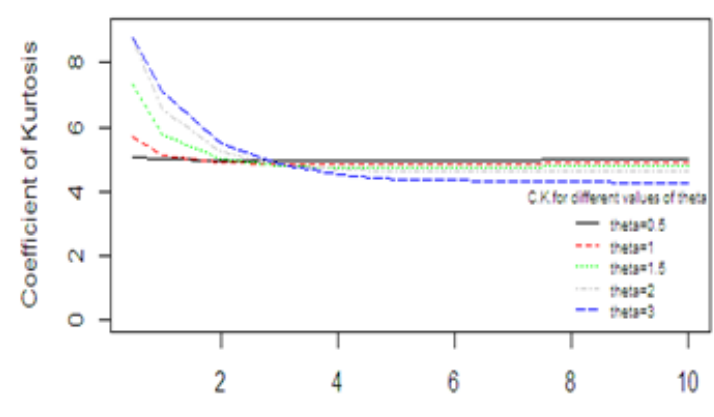

alpha
Graphs of C.V, $\sqrt{\beta_{1}}, \beta_{2}$ and $\gamma$ of QSD for varying values of the parameters $\theta$ and $\alpha$ have been presented in Figure 3.
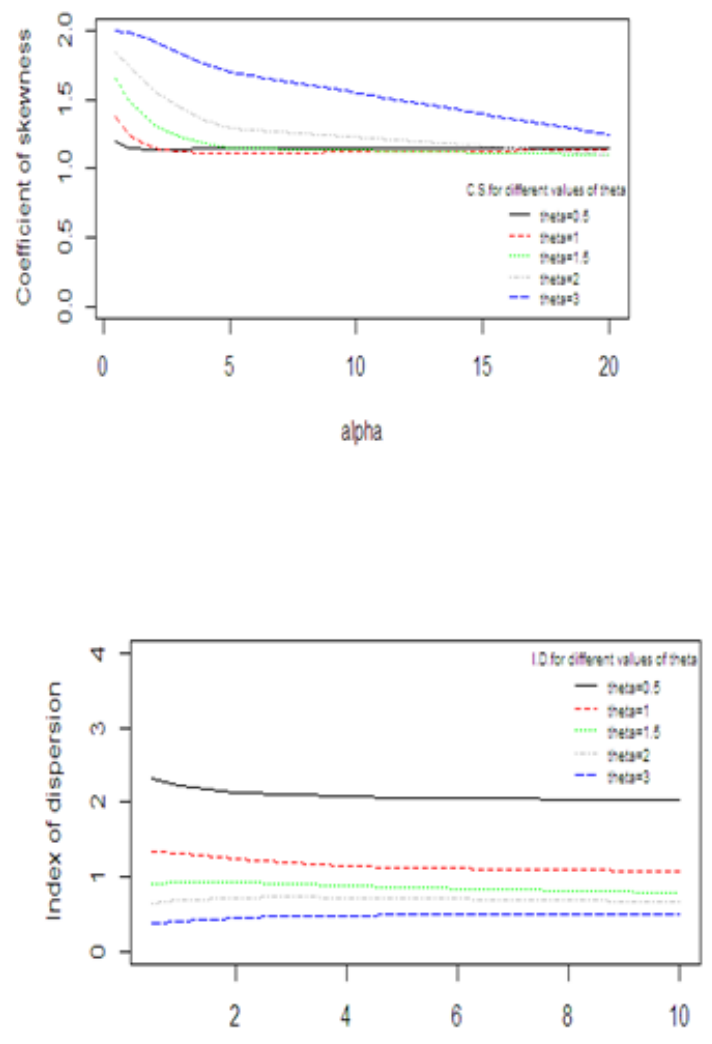

alpha

Figure 3 Graphs of C.V, $\sqrt{\beta_{1}}, \beta_{2}$ and $\gamma$ of QSD for varying values of the parameter $\theta$ and $\alpha$.

\section{Hazard rate function and mean residual life function}

Suppose $X$ be a continuous random variable with pdf $f(x)$ and cdf $X$. The hazard rate function (also known as the failure rate function) and the mean residual life function of $X$ are respectively defined as

$$
h(x)=\lim _{\Delta x \rightarrow 0} \frac{P(X<x+\Delta x \mid X>x)}{\Delta x}=\frac{f(x)}{1-F(x)}
$$

$$
\text { And } m(x)=E[X-x \mid X>x]=\frac{1}{1-F(x)} \int_{x}^{\infty}[1-F(t)] d t
$$

The corresponding hazard rate function $h(x)$, and the mean residual life function $m(x)$ of QSD are thus obtained as

$$
h(x)=\frac{\theta^{3}\left(\theta+x+\alpha x^{2}\right)}{\alpha \theta^{2} x^{2}+\theta(\theta+2 \alpha) x+\left(\theta^{3}+\theta+2 \alpha\right)}
$$

and

$$
\begin{gathered}
m(x)=\frac{1}{\left[\alpha \theta^{2} x^{2}+\theta(\theta+2 \alpha) x+\left(\theta^{3}+\theta+2 \alpha\right)\right] e^{-\theta x} \int_{x}^{\infty}\left[\begin{array}{l}
\alpha \theta^{2} t^{2}+\theta(\theta+2 \alpha) t \\
+\left(\theta^{3}+\theta+2 \alpha\right)
\end{array}\right] e^{-\theta t} d t} \\
=\frac{\alpha \theta^{2} x^{2}+\theta(\theta+4 \alpha) x+\left(\theta^{3}+2 \theta+6 \alpha\right)}{\theta\left[\alpha \theta^{2} x^{2}+\theta(\theta+2 \alpha) x+\left(\theta^{3}+\theta+2 \alpha\right)\right]}
\end{gathered}
$$


It can be easily verified that $h(0)=\frac{\theta^{4}}{\theta^{3}+\theta+2 \alpha}=f(0)$ and $m(0)=\frac{\theta^{3}+2 \theta+6 \alpha}{\theta\left(\theta^{3}+\theta+2 \alpha\right)}=\mu_{1}^{\prime}$
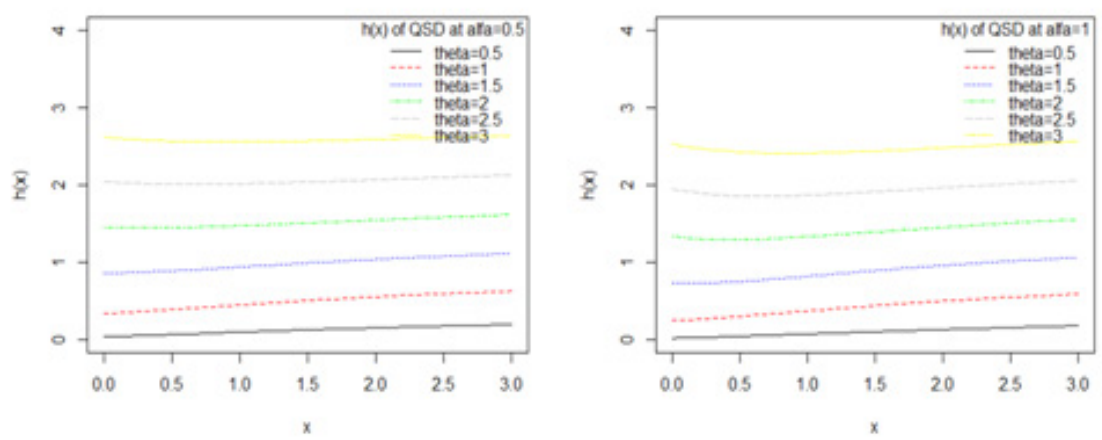

Figure 4 Graphs of $h(x)$ of QSD for varying values of parameters $\theta$ and $\alpha$.
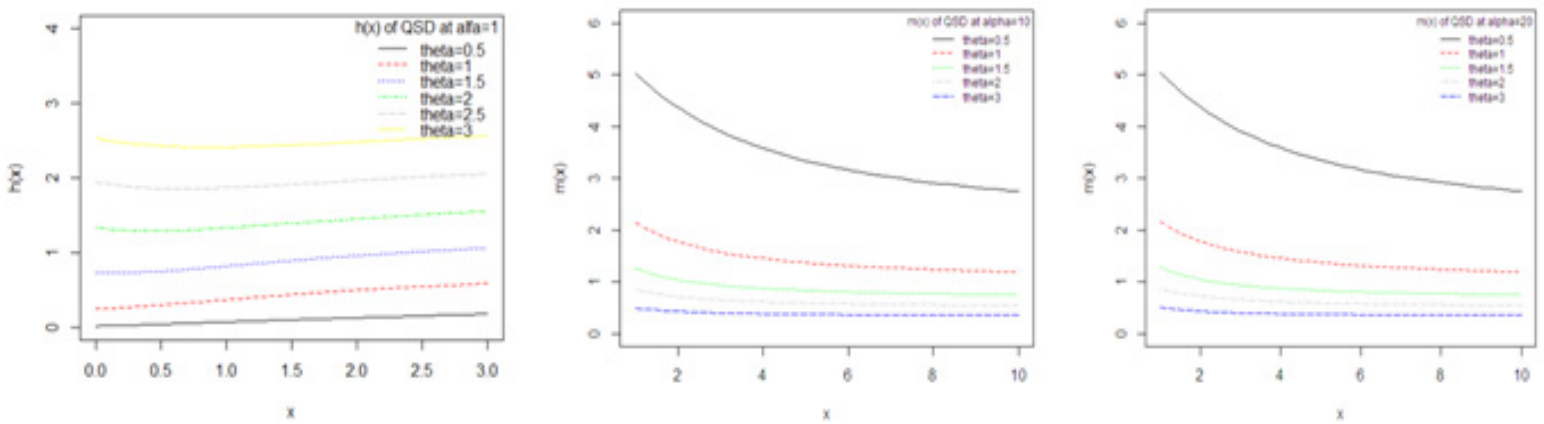

Figure 5 Graphs of $m(x)$ of QSD for varying values of parameters $\theta$ and $\alpha$.

\section{Stochastic orderings}

Stochastic ordering of positive continuous random variables is an important tool for judging their comparative behavior. A random variable $X$ is said to be smaller than a random variable $Y$ in the

i. $\quad$ stochastic order $\left(X \leq_{s t} Y\right)$ if $F_{X}(x) \geq F_{Y}(x)$ for all $x$

ii. hazard rate order $\left(X \leq_{s t} Y\right)$ if $h_{X}(x) \geq h_{Y}(x)$ for all $x$

iii. mean residual life order $\left(X \leq_{s t} Y\right)$ if $m_{X}(x) \leq m_{Y}(x)$ for all $x$

iv. likelihood ratio order $f_{Y}(x)$ if $\frac{f_{X}(x)}{f_{Y}(x)}$ decreases in $x$.

The following results due to Shaked and Shanthikumar ${ }^{4}$ are well known for establishing stochastic ordering of distributions

$$
X \leq_{l r} Y \Rightarrow X \leq_{h r} Y \Rightarrow X \leq_{m r l} Y
$$

The nature and behavior of $h(x)$ and $m(x)$ of QSD for varying values of parameters $\theta$ and $\alpha$ have been shown graphically in Figures $4 \& 5$. It is obvious that $h(x)$ of QSD is monotonically increasing whereas $h(x)$ is monotonically decreasing.

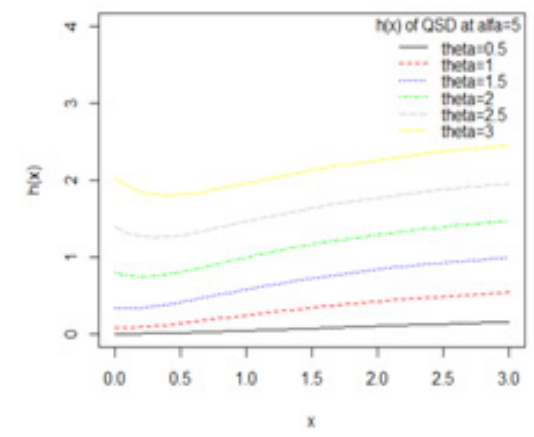


Now

$$
\ln \frac{f_{X}\left(x ; \theta_{1}, \alpha_{1}\right)}{f_{Y}\left(x ; \theta_{2}, \alpha_{2}\right)}=\log \left[\frac{\theta_{1}^{3}\left(\theta_{2}^{3}+\theta_{2}+2 \alpha_{2}\right)}{\theta_{2}^{3}\left(\theta_{1}^{3}+\theta_{1}+2 \alpha_{1}\right)}\right]+\ln \left(\frac{\theta_{1}+x+\alpha_{1} x^{2}}{\theta_{2}+x+\alpha_{2} x^{2}}\right)-\left(\theta_{1}-\theta_{2}\right) x
$$

This gives

$$
\frac{d}{d x}\left\{\ln \frac{f_{X}\left(x ; \theta_{1}, \alpha_{1}\right)}{f_{Y}\left(x ; \theta_{2}, \alpha_{2}\right)}\right\}=\frac{\left(\theta_{2}-\theta_{1}\right)+\left(\alpha_{2}-\alpha_{1}\right)+2\left(\alpha_{1} \theta_{2}-\alpha_{2} \theta_{1}\right) x+2\left(\alpha_{1}-\alpha_{2}\right) x^{2}}{\left(\theta_{1}+x+\alpha_{1} x^{2}\right)\left(\theta_{2}+x+\alpha_{2} x^{2}\right)}-\left(\theta_{1}-\theta_{2}\right)
$$

Thus if $\alpha_{1}=\alpha_{2}$ and $\theta_{1}>\theta_{2}$ or $\theta_{1}=\theta_{2}$ and $\alpha_{1}<\alpha_{2}$,

$\frac{d}{d x} \ln \frac{f_{X}\left(x ; \theta_{1}, \alpha_{1}\right)}{f_{Y}\left(x ; \theta_{2}, \alpha_{2}\right)}<0$. This means that $X \leq_{h r} Y$ and hence

$X \leq_{h r} Y, X \leq_{s t} Y$ and $X \leq_{s t} Y$.

\section{Mean deviations from the mean and the median}

The amount of scatter in a population is measured to some extent by the totality of deviations usually from mean and median. These are known as the mean deviation about the mean and the mean deviation about the median defined by

$$
\text { and } \delta_{2}(X)=\int_{0}^{\infty}|x-M| f(x) d x \text {, respectively, where } \mu=E(X)
$$

and $M=\operatorname{Median}(X)$. The measures $\delta_{1}(X)$ and $\delta_{2}(X)$ can be calculated using the following simplified relationships

$$
\delta_{1}(X)=\int_{0}^{\mu}(\mu-x) f(x) d x+\int_{\mu}^{\infty}(x-\mu) f(x) d x
$$

Using p.d.f. (2.1) and expression for the mean of QSD, we get

$$
\begin{aligned}
& \int_{0}^{\mu} x f(x) d x=\mu-\frac{\left.\left\{N N^{3} N^{3}+S^{2} x+3\right)^{2}+\left({ }^{3}+2+6\right)+\left({ }^{3}+2+6\right)\right\} e^{-\theta \mu}}{\theta\left(\theta^{3}+\theta+2 \alpha\right)} \\
& \int_{0}^{M} x f(x) d x=\mu-\frac{\left\{\alpha \theta^{3} M^{3}+\theta^{2}(\theta+3 \alpha) M^{2}+\theta\left(\theta^{3}+2 \theta+6 \alpha\right) M+\left(\theta^{3}+2 \theta+6 \alpha\right)\right\} e^{-\theta M}}{\theta\left(\theta^{3}+\theta+2 \alpha\right)}
\end{aligned}
$$

Using expressions from (6.1), (6.2), (6.3), and (6.4), the mean deviation about mean, $\delta_{1}(X)$ and the mean deviation about median, $\delta_{2}(X)$ of QSD are finally obtained as

$$
\begin{gathered}
\delta_{1}(X)=\frac{2\left\{\alpha \theta^{2} \mu^{2}+\theta(\theta+4 \alpha) \mu+\left(\theta^{3}+2 \theta+6 \alpha\right)\right\} e^{-\theta \mu}}{\theta\left(\theta^{3}+\theta+2 \alpha\right)} \\
\delta_{2}(X)=\frac{2\left\{\alpha \theta^{3} M^{3}+\theta^{2}(\theta+3 \alpha) M^{2}+\theta\left(\theta^{3}+2 \theta+6 \alpha\right) M+\left(\theta^{3}+2 \theta+6 \alpha\right)\right\} e^{-\theta M}}{\theta\left(\theta^{3}+\theta+2 \alpha\right)}-\mu
\end{gathered}
$$




\section{Bonferroni and lorenz curves}

The Bonferroni and Lorenz curves ${ }^{5}$ and Bonferroni and Gini indices have applications not only in economics to study income and poverty, but also in other fields like reliability, demography, insurance and medicine. The Bonferroni and Lorenz curves are defined as

$$
\begin{aligned}
& B(p)=\frac{1}{p \mu} \int_{0}^{q} x f(x) d x=\frac{1}{p \mu}\left[\int_{0}^{\infty} x f(x) d x-\int_{q}^{\infty} x f(x) d x\right]=\frac{1}{p \mu}\left[\begin{array}{c}
\left.\mu-\int_{q}^{\infty} x f(x) d x\right] \\
\text { and } L(p)=\frac{1}{\mu} \int_{0}^{q} x f(x) d x=\frac{1}{\mu}\left[\int_{0}^{\infty} x f(x) d x-\int_{q}^{\infty} x f(x) d x\right. \\
0
\end{array}\right]=\frac{1}{\mu}\left[\begin{array}{c}
\left.\mu-\int_{q}^{\infty} x f(x) d x\right] \\
q
\end{array}\right]
\end{aligned}
$$

Respectively or equivalently

$$
\begin{array}{r}
B(p)=\frac{1}{p \mu} \int_{0}^{p} F^{-1}(x) d x \\
\text { and } L(p)=\frac{1}{\mu} \int_{0}^{p} F^{-1}(x) d x
\end{array}
$$

Respectively, where $\mu=E(X)$ and $q=F^{-1}(p)$.
The Bonferroni and Gini indices are thus defined as

$$
\begin{aligned}
& \qquad B=1-\int_{0}^{1} B(p) d p \\
& \text { and } G=1-2 \int_{0}^{1} L(p) d p \\
& \text { respectively. }
\end{aligned}
$$

Using p.d.f. of QSD (2.1), we get

$$
\int_{q}^{\infty} x f(x) d x=\frac{\left\{\alpha \theta^{3} q^{3}+\theta^{2}(\theta+3 \alpha) q^{2}+\theta\left(\theta^{3}+2 \theta+6 \alpha\right) q+\left(\theta^{3}+2 \theta+6 \alpha\right)\right\} e^{-\theta q}}{\theta\left(\theta^{3}+\theta+2 \alpha\right)}
$$

Now using equation (7.7) in (7.1) and (7.2), we get

$$
B(p)=\frac{1}{p}\left[1-\frac{\left\{\alpha \theta^{3} q^{3}+\theta^{2}(\theta+3 \alpha) q^{2}+\theta\left(\theta^{3}+2 \theta+6 \alpha\right) q+\left(\theta^{3}+2 \theta+6 \alpha\right)\right\} e^{-\theta q}}{\theta^{3}+2 \theta+6 \alpha}\right]
$$

and

$$
L(p)=1-\frac{\left\{\alpha \theta^{3} q^{3}+\theta^{2}(\theta+3 \alpha) q^{2}+\theta\left(\theta^{3}+2 \theta+6 \alpha\right) q+\left(\theta^{3}+2 \theta+6 \alpha\right)\right\} e^{-\theta q}}{\theta^{3}+2 \theta+6 \alpha}
$$

Now using equations (7.8) and (7.9) in (7.5) and (7.6), the Bonferroni and Gini indices of QSD are thus obtained as

$$
\begin{gathered}
B=1-\frac{\left\{\alpha \theta^{3} q^{3}+\theta^{2}(\theta+3 \alpha) q^{2}+\theta\left(\theta^{3}+2 \theta+6 \alpha\right) q+\left(\theta^{3}+2 \theta+6 \alpha\right)\right\} e^{-\theta q}}{\theta^{3}+2 \theta+6 \alpha} \\
G=\frac{2\left\{\alpha \theta^{3} q^{3}+\theta^{2}(\theta+3 \alpha) q^{2}+\theta\left(\theta^{3}+2 \theta+6 \alpha\right) q+\left(\theta^{3}+2 \theta+6 \alpha\right)\right\} e^{-\theta q}}{\theta^{3}+2 \theta+6 \alpha}-1
\end{gathered}
$$

\section{Stress-strength reliability}

The stress- strength reliability describes the life of a component which has random strength $X$ that is subjected to a random stress $Y$. When the stress applied to it exceeds the strength, the component fails instantly and the component will function satisfactorily till $X>Y$. Therefore, $R=P(Y<X)$ is a measure of component reliability and in statistical literature it is known as stress-strength parameter. It has wide applications in almost all areas of knowledge especially in engineering such as structures, deterioration of rocket motors, static fatigue of ceramic components, aging of concrete pressure vessels etc. Let $X$ and $Y$ be independent streng th and stress random variables having QSD (2.1) with parameter $\left(\theta_{1}, \alpha_{1}\right)$ and $\left(\theta_{2}, \alpha_{2}\right)$ respectively. Then the stress-strength reliability $R$ of QSD (2.1) can be obtained as

$$
\begin{aligned}
R= & P(Y<X)=\int_{0}^{\infty} P(Y<X \mid X=x) f_{X}(x) d x \\
& =\int_{0}^{\infty} f\left(x ; \theta_{1}, \alpha_{1}\right) F\left(x ; \theta_{2}, \alpha_{2}\right) d x
\end{aligned}
$$




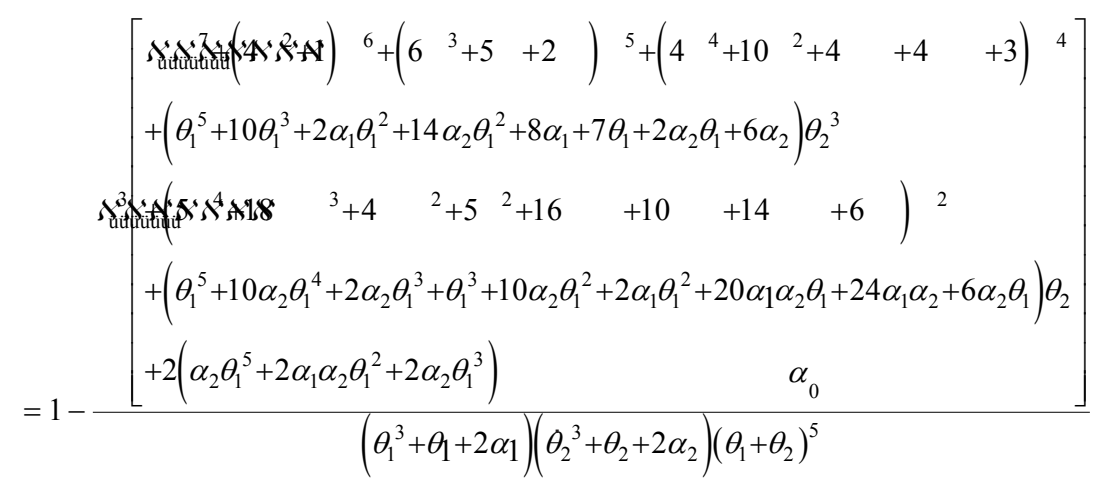

It can be easily verified that at $\alpha_{1}=0$ and $\alpha_{2}=0$, the above expression reduces to the corresponding expression for Shanker distribution introduced by Shanker. ${ }^{1}$

\section{Maximum likelihood estimation of parameters}

Let $\left(x_{1}, x_{2}, x_{3}, \ldots, x_{n}\right)$ be a random sample of size $n$ from QSD (2.1)). The likelihood function, $L$ of (2.1) is given by

$$
L=\left(\frac{\theta^{3}}{\theta^{3}+\theta+2 \alpha}\right)^{n} \prod_{i=1}^{n}\left(\theta+x_{i}+\alpha x_{i}^{2}\right) e^{-n \theta \bar{x}}
$$

The natural $\log$ likelihood function is thus obtained as

$$
\ln L=n \ln \left(\frac{\theta^{3}}{\theta^{3}+\theta+2 \alpha}\right)+\sum_{i=1}^{n} \ln \left(\theta+x_{i}+\alpha x_{i}^{2}\right)-n \theta \bar{x}
$$

The maximum likelihood estimates (MLE) $(\hat{\theta}, \hat{\alpha})$ of $(\theta, \alpha)$ are then the solutions of the following non-linear equations

$$
\frac{\partial \ln L}{\partial \theta}=\frac{3 n}{\theta}-\frac{n\left(3 \theta^{2}+1\right)}{\theta^{3}+\theta+2 \alpha}+\sum_{i=1}^{n} \frac{1}{\theta+x_{i}+\alpha x_{i}^{2}}-n \bar{x}=0
$$

where $\bar{x}$ is the sample mean.

These two natural log likelihood equations do not seem to be solved directly because they are not in closed forms. However, the Fisher's scoring method can be applied to solve these equations. For we have

\section{Data set I}

This data set is the strength data of glass of the aircraft window reported by Fuller et al., ${ }^{6}$

$\begin{array}{lllllllllllll}18.83 & 20.8 & 21.657 & 23.03 & 23.23 & 24.05 & 24.321 & 25.5 & 25.52 & 25.8 & 26.69 & 26.77 & 26.78 \\ 27.05 & 27.67 & 29.9 & 31.11 & 33.2 & 33.73 & 33.76 & 33.89 & 34.76 & 35.75 & 35.91 & 36.98 & 37.08 \\ 37.09 & 39.58 & 44.045 & 45.29 & 45.381 & & & & & & & & \end{array}$

$$
\frac{\partial^{2} \ln L}{\partial \theta^{2}}=-\frac{3 n}{\theta^{2}}+\frac{n\left(3 \theta^{4}-6 \theta^{3}+5 \theta^{2}-12 \theta \alpha+1\right) \alpha^{2}}{\left(\theta^{3}+\theta+2 \alpha\right)^{2}}-\sum_{i=1}^{n} \frac{1}{\left(\theta+x_{i}+\alpha x_{i}^{2}\right)^{2}}
$$$$
\frac{\partial^{2} \ln L}{\partial \theta \partial \alpha}=\frac{2 n\left(3 \theta^{2}+1\right)}{\left(\theta^{3}+\theta+2 \alpha\right)^{2}}-\sum_{i=1}^{n} \frac{x_{i}^{2}}{\left(\theta+x_{i}+\alpha x_{i}^{2}\right)^{2}}
$$$$
\frac{\partial^{2} \ln L}{\partial \alpha^{2}}=\frac{4 n}{\left(\theta^{3}+\theta+2 \alpha\right)^{2}}-\sum_{i=1}^{n} \frac{x_{i}^{4}}{\left(\theta+x_{i}+\alpha x_{i}^{2}\right)^{2}}
$$

The solution of following equations gives MLE's $(\hat{\theta}, \hat{\alpha})$ of $(\theta, \alpha)$ of QSD

$$
\left[\begin{array}{ll}
\frac{\partial^{2} \ln L}{\partial \theta^{2}} & \frac{\partial^{2} \ln L}{\partial \theta \partial \alpha} \\
\frac{\partial^{2} \ln L}{\partial \theta \partial \alpha} & \frac{\partial^{2} \ln L}{\partial \alpha^{2}}
\end{array}\right]_{\substack{\hat{\theta}=\theta_{0} \\
\hat{\alpha}=\alpha_{0}}}\left[\begin{array}{c}
\hat{\theta}-\theta_{0} \\
\hat{\alpha}-\alpha_{0}
\end{array}\right]=\left[\begin{array}{c}
\frac{\partial \ln L}{\partial \theta} \\
\frac{\partial \ln L}{\partial \alpha}
\end{array}\right]_{\substack{\hat{\theta}=\theta_{0} \\
\hat{\alpha}}}
$$

where $\theta_{0}$ and are the initial values of $\theta$ and $\alpha$, respectively. These equations are solved iteratively till sufficiently close values of $\hat{\theta}$ and $\hat{\alpha}$ are obtained.

\section{Data analysis}

In this section, the goodness of fit of QSD has been discussed with two real lifetime data sets from engineering and the fit has been compared with one parameter exponential, Lindley and Shanker distributions. The following two data sets have been considered. 


\section{Data set 2}

The following data represent the tensile strength, measured in GPa, of 69 carbon fibers tested under tension at gauge lengths of $20 \mathrm{~mm}$, Bader and Priest. ${ }^{7}$

$\begin{array}{lllllllllllll}1.312 & 1.314 & 1.479 & 1.552 & 1.700 & 1.803 & 1.861 & 1.865 & 1.944 & 1.958 & 1.966 & 1.997 & 2.006 \\ & 2.021 & 2.027 & 2.055 & 2.063 & 2.098 & 2.140 & 2.179 & 2.224 & 2.240 & 2.253 & 2.270 & 2.272 \\ & 2.274 & 2.301 & 2.301 & 2.359 & 2.382 & 2.382 & 2.426 & 2.434 & 2.435 & 2.478 & 2.490 & 2.511 \\ 2.514 & 2.535 & 2.554 & 2.566 & 2.570 & 2.586 & 2.629 & 2.633 & 2.642 & 2.648 & 2.684 & 2.697 \\ & 2.726 & 2.770 & 2.773 & 2.800 & 2.809 & 2.818 & 2.821 & 2.848 & 2.880 & 2.954 & 3.012 & 3.067 \\ & 3.084 & 3.090 & 3.096 & 3.128 & 3.233 & 3.433 & 3.585 & 3.585 & & & & \end{array}$

In order to compare the considered distributions, values of $-2 \ln L$, AIC(Akaike Information Criterion) and K-S Statistic ( KolmogorovSmirnov Statistic) for the data sets have been computed and presented in Table 1. The formula for AIC and K-S Statistic is defined as follow:

Table I MLE's, $-2 \ln L$, standard error,AIC, and K-S statistic of the fitted distributions of data sets I and 2

\begin{tabular}{|c|c|c|c|c|c|c|}
\hline Data sets & Distributions & ML estimates & Standard errors & $-2 \ln L$ & AIC & K-S statistic \\
\hline \multirow{6}{*}{ I } & \multirow{3}{*}{ QSD } & $\hat{\theta}=0.097330$ & 0.0101017 & \multirow{3}{*}{240.53} & \multirow{3}{*}{244.53} & \multirow{3}{*}{0.298} \\
\hline & & & & & & \\
\hline & & $\hat{\alpha}=13.623065$ & 52.81378 & & & \\
\hline & Shanker & $\hat{\theta}=0.6471636$ & 0.0082 & 252.35 & 254.35 & 0.358 \\
\hline & Lindley & $\hat{\theta}=0.062990$ & 0.008 & 253.98 & 255.98 & 0.365 \\
\hline & Exponential & $\hat{\theta}=0.032449$ & 0.005822 & 274.53 & 276.53 & 0.458 \\
\hline \multirow{5}{*}{2} & QSD & $\hat{\theta}=1.20552$ & $0.08386 I$ & \multirow[t]{2}{*}{186.78} & \multirow[t]{2}{*}{190.78} & \multirow{2}{*}{0.314} \\
\hline & & $\hat{\alpha}=49.73844$ & 34.58363 & & & \\
\hline & Shanker & $\hat{\theta}=0.658030$ & 0.052373 & 233 & 235 & 0.369 \\
\hline & Lindley & $\hat{\theta}=0.65450$ & 0.058031 & 238.38 & 240.38 & 0.401 \\
\hline & Exponential & $\hat{\theta}=0.407942$ & 0.04911 & 261.73 & 263.73 & 0.448 \\
\hline
\end{tabular}

$A I C=-2 \ln L+2 k$ and $K-S=\operatorname{Sup}\left|F_{n}(x)-F_{0}(x)\right|$, where $k=$ number of parameters, $n=$ sample size, $F_{n}(x)$ is the empirical distribution function and $F_{0}(x)$ is the theoretical cumulative distribution function. The best distribution corresponds to lower values of $-2 \ln L$ , AIC and K-S statistic. It can be easily seen from table 1 that the QSD gives better fit than one parameter exponential, Lindley and Shanker distributions and hence it can be considered as an important distribution for modeling lifetime data from engineering.

\section{Concluding remarks}

A two-parameter quasi Shanker distribution (QSD), of which one parameter Shanker distribution introduced by Shanker ${ }^{1}$ is a particular case, has been suggested and investigated. Its mathematical properties including moments, coefficient of variation, skewness, kurtosis, index of dispersion, hazard rate function, mean residual life function, stochastic ordering, mean deviations, Bonferroni and Lorenz curves, and stress-strength reliability have been discussed. For estimating its parameters method of maximum likelihood estimation has been discussed. Finally, two numerical examples of real lifetime data sets has been presented to test the goodness of fit of QSD over exponential, Lindley and Shanker distributions and the fit by QSD has been quite satisfactory. Therefore, QSD can be recommended as an important two-parameter lifetime distribution.

\section{Acknowledgements}

None.

\section{Conflict of interest}

None.

\section{References}

1. Shanker R. Shanker distribution and Its Applications. International Journal of Statistics and Applications. 2015;5(6):338-348. 
2. Lindley DV. Fiducial distributions and Bayes' theorem, Journal of the Royal Statistical Society. Series B. 1958;20(1):102-107.

3. Shanker R. The discrete Poisson-Shanker distribution. Jacobs Journal of Biostatistics. 2016;2(2):41-21.

4. Shaked M, Shanthikumar JG (1994) Stochastic Orders and Their Applications. Academic Press. New York.
5. Bonferroni CE. Elementi di Statistca generale, Seeber, Firenze. 1930.

6. Fuller EJ, Frieman S, Quinn J, et al. Fracture mechanics approach to the design of glass aircraft windows: A case study. SPIE Proc. 1994;2286:419-430.

7. Bader MG, Priest AM. Statistical aspects of fiber and bundle strength in hybrid composites. In; hayashi T, Kawata K Umekawa S (Eds.), Progressin Science in Engineering Composites, ICCM-IV, Tokyo. 1982;1129-1136. 